EPJ manuscript No.

(will be inserted by the editor)

\title{
Rare Charm and B Decays at CDF
}

\author{
Julia Thom ${ }^{1}$ (on behalf of the CDF Collaboration) \\ Fermi National Accelerator Laboratory, P.O.Box 500, Batavia, IL 60501-0500, USA \\ Received: date / Revised version: date
}

\begin{abstract}
We present results on rare charm and B decays using $65 p b^{-1}$ of data taken with the CDF detector in Run II. Three results are discussed, a measurement of the relative branching ratios $\Gamma\left(D^{0} \rightarrow\right.$ $\left.K^{+} K^{-}\right) / \Gamma\left(D^{0} \rightarrow K \pi\right)$ and $\Gamma\left(D^{0} \rightarrow \pi^{+} \pi^{-}\right) / \Gamma\left(D^{0} \rightarrow K \pi\right)$ and the direct CP-violating decay rate asymmetry, and a limit on the branching ratio of the FCNC decay $D^{0} \rightarrow \mu^{+} \mu^{-}$. We also discuss the prospects for the search for $B_{s}^{0} \rightarrow \mu^{+} \mu^{-}$decays.
\end{abstract}

PACS. PACS-key discribing text of that key - PACS-key discribing text of that key

\section{Introduction}

After a 5 year shutdown with a major detector and accelerator upgrade, CDF restarted data taking in March 2001. Several detector systems have been replaced, such as the extended vertex detector. In addition, CDF II has a new trigger and data aquisition system. For the first time in an hadronic environment, it is possible to trigger on tracks with a large impact parameter at the hardware level (Silicon Vertex Trigger SVT), see Fig.1 for the SVT impact parameter distribution. The total width is given by the effective measurement resolution of $35 \mu \mathrm{m}$ convoluted with the Tevatron intrinsic beam size of $33 \mu \mathrm{m}$.

Among the interesting physics program of CDF is the study of rare decays of charm and $\mathrm{b}$ mesons, which is an important tool to understand the Standard Model, and a potential window for physics beyond it. Processes like the FCNC decays $D^{0} \rightarrow \mu^{+} \mu^{-}$or $B_{s}^{0} \rightarrow \mu^{+} \mu^{-}$are highly suppressed in the Standard Model, as are the direct CPviolating decay rate asymmetries of Cabibbo suppressed $D^{0}$ decays. New physics can significantly enhance these processes, for example the presence of SUSY in $\mathrm{SO}(10)$ models predicts large enhancements to $B_{s}^{0} \rightarrow \mu^{+} \mu^{-}$and would make them observable in the Run II of the Tevatron.

\section{CP Asymmetries and Decay Rate Ratios of Cabibbo suppressed $D^{0}$ decays}

For this analysis we measure the relative branching ratios $\Gamma\left(D^{0} \rightarrow K^{+} K^{-}\right) / \Gamma\left(D^{0} \rightarrow K \pi\right)$ and $\Gamma\left(D^{0} \rightarrow \pi^{+} \pi^{-}\right) / \Gamma\left(D^{0} \rightarrow K \pi\right)$, and perform a measurement of the direct $\mathrm{CP}$-violating decay rate asymmetry.

Send offprint requests to:

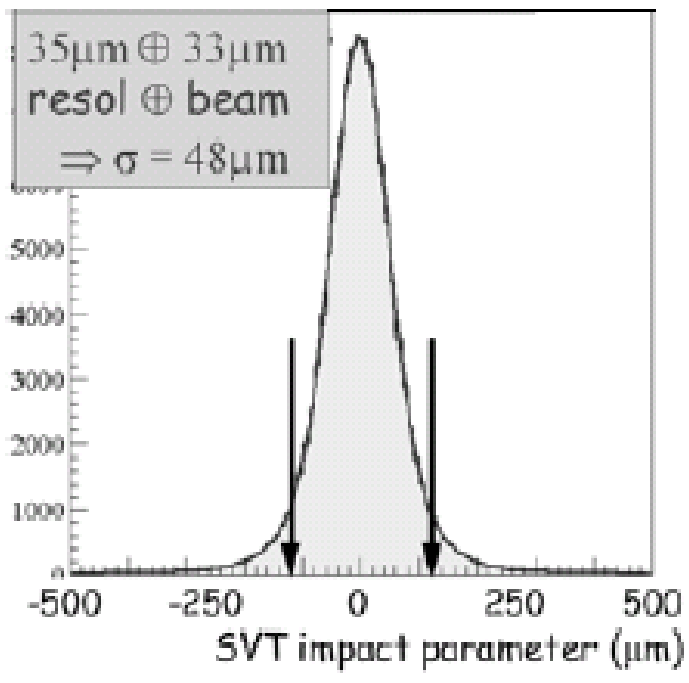

Fig. 1. The SVT impact parameter distribution. The arrows indicate the cut at $\pm 120 \mu m$ to reject primary tracks.

The Standard Model predictions for the rate of CP violation in the charm sector is small, ranging from $0.1 \%$ to $1 \%$ (see for example [1]). Observation of $\mathrm{CP}$ violation above the $1 \%$ level would be a strong indication for physics beyond the Standard Model.

$D^{0} \rightarrow K \pi, D^{0} \rightarrow K^{+} K^{-}$and $D^{0} \rightarrow \pi^{+} \pi^{-}$candidates are reconstructed using two tracks, selected by the SVT. The latter requires two opposite charged tracks with transverse momentum greater than $2 \mathrm{GeV} / \mathrm{c}$ and impact parameter between $120 \mu \mathrm{m}$ and $1 \mathrm{~mm}$. The reconstructed $D^{0}$ candidates are then combined with a soft pion track to reconstruct the final $D^{* \pm} \rightarrow D^{0} \pi^{ \pm}$candidates. The charge of the soft pion is required to match the charge of the pion from the $D^{0}$ decay. No particle identification has been used in the analysis. About $100000 D^{0} \rightarrow K \pi, 8000$ 
$D^{0} \rightarrow K^{+} K^{-}$and $4000 D^{0} \rightarrow \pi \pi$ candidates (tagged with $D^{*}$ ) pass our selection cuts: $D^{0}$ impact parameter less than $100 \mu \mathrm{m}, 2$-dimensional $D^{0}$ decay length in the transverse plane $>350 \mu m, \Delta M=M\left(D^{*}\right)-M\left(D^{0}\right)$ inside a $3 \sigma$ window $\left(\sigma \sim 0.6 \mathrm{MeV} / \mathrm{c}^{2}\right)$ around the expected value, and the two $D^{0}$ tracks are required to have opposite charge. We determine the relative branching ratio as

$$
\frac{\Gamma\left(D^{0} \rightarrow K K(\pi \pi)\right)}{\Gamma\left(D^{0} \rightarrow K \pi\right)}=\frac{N_{K K(\pi \pi)}}{N_{K \pi}} \frac{\epsilon_{K \pi}}{\epsilon_{K K(\pi \pi)}}
$$

where $N_{h^{ \pm} h \mp}$ is the number of $D^{0}$ decaying in the appropriate mode and $\epsilon_{h^{ \pm}} h^{\mp}$ is the overall geometric and kinematic acceptance for each of the decay modes. This includes both trigger efficiency and offline reconstruction efficiency, and is estimated including realistic emulation of the SVT trigger, the effects of nuclear interactions and decay in flight of kaons and pions and time dependent detector inefficiencies. We use the same procedure to search for the direct CP asymmetries $A_{C P}=\frac{\Gamma\left(D^{0} \rightarrow f\right)-\Gamma\left(\overline{D^{0}} \rightarrow f\right)}{\Gamma\left(D^{0} \rightarrow f\right)+\Gamma\left(\overline{D^{0}} \rightarrow f\right)}$, where $\mathrm{f}$ can be $K^{+} K^{-}$or $\pi^{+} \pi^{-}$. The charge of the slow pion from the $D^{*}$ decay serves as an unbiased tag of the $D^{0}$ flavor since charm quarks are mainly produced in quarkantiquark pairs at the Tevatron, and fragmentation and $D^{*}$ decay are strong processes which conserve CP. We correct for the intrinsic charge asymmetry of the CDF detector studying this effect on samples of unbiased tracks and testing any possible residual effect after the correction on independent samples of meson decays where CPasymmetry is not expected. The residual asymmetry is $0.38 \pm 0.59 \%$, which is the largest contribution to the systematic error. This will improve with larger statistics.

We obtain the following relative branching fractions:

$$
\begin{aligned}
& \frac{\Gamma\left(D^{0} \rightarrow K^{+} K^{-}\right)}{\Gamma\left(D^{0} \rightarrow K \pi\right)}=9.38 \pm 0.18(\text { stat }) \pm 0.10(\text { syst }) \% \\
& \frac{\Gamma\left(D^{0} \rightarrow \pi^{ \pm} \pi^{ \pm}\right)}{\Gamma\left(D^{0} \rightarrow K \pi\right)}=3.686 \pm 0.076(\text { stat }) \pm 0.036(\text { syst }) \%
\end{aligned}
$$

and for the direct $\mathrm{CP}$ asymmetries we find

$$
\begin{aligned}
A\left(D^{0} \rightarrow K^{+} K^{-}\right) & =2.0 \pm 1.7(\text { stat }) \pm 0.6(\text { syst }) \% \\
A\left(D^{0} \rightarrow \pi^{+} \pi^{-}\right) & =3.0 \pm 1.9(\text { stat }) \pm 0.6(\text { syst }) \%
\end{aligned}
$$

These results compare favorably with the best single experiments (see Focus [2] and CLEO[3] results).

\section{Search for the FCNC Decay $D^{0} \rightarrow \mu^{+} \mu^{-}$}

The FCNC Decay $D^{0} \rightarrow \mu^{+} \mu^{-}$is highly suppressed in the Standard Model $\left(B R\left(D^{0} \rightarrow \mu^{+} \mu^{-}\right) \sim 10^{-13}\right)$, but new physics can enhance this mode (see [4]). Similar to the analysis of $D^{0}$ decays described above, we use two tracks selected by the SVT. We normalize to the $D^{0} \rightarrow \pi^{+} \pi^{-}$ mode to cancel acceptance and common trigger efficiencies. The limit on the branching ratio is computed as

$$
\begin{aligned}
& B R\left(D^{0} \rightarrow \mu^{+} \mu^{-}\right)< \\
& \frac{N_{C L}\left(D^{0} \rightarrow \mu^{+} \mu^{-}\right)}{N\left(D^{0} \rightarrow \pi^{+} \pi^{-}\right)} \frac{\epsilon\left(D^{0} \rightarrow \pi^{+} \pi^{-}\right)}{\epsilon\left(D^{0} \rightarrow \mu^{+} \mu^{-}\right)} B R\left(D^{0} \rightarrow \pi^{+} \pi^{-}\right)
\end{aligned}
$$

\section{CDF Run II Preliminary}

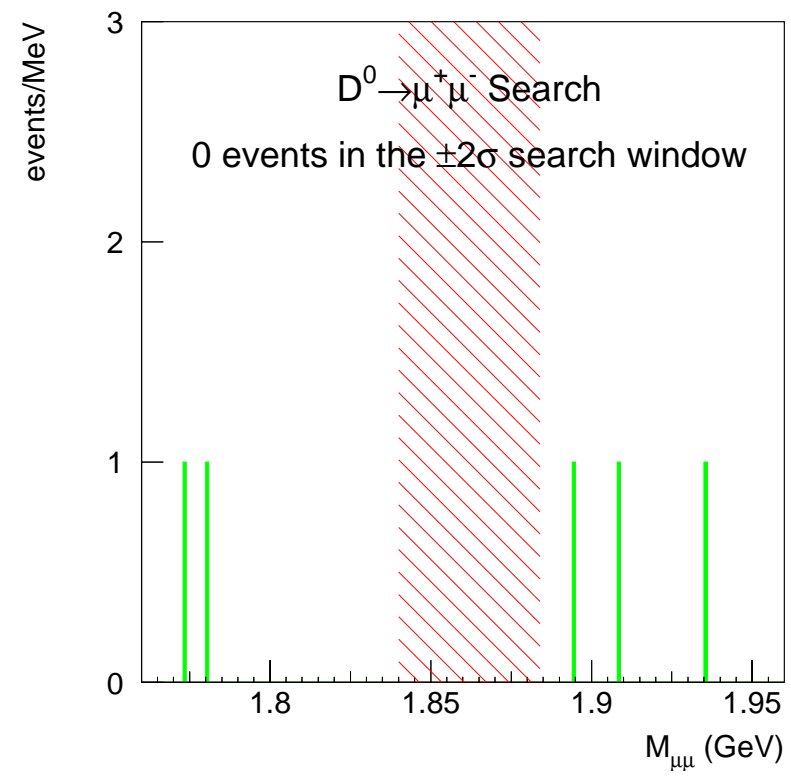

Fig. 2. Event counts in the search window for $D^{0} \rightarrow \mu^{+} \mu^{-}$ events.

where $N_{C L}\left(D^{0} \rightarrow \mu^{+} \mu^{-}\right)$is the $90 \%$ C.L.upper limit on the number of observed decays, $N\left(D^{0} \rightarrow \pi^{+} \pi^{-}\right)$is the number of $D^{0}$ reconstructed in the normalization mode, $\frac{\epsilon\left(D^{0} \rightarrow \pi^{+} \pi^{-}\right)}{\epsilon\left(D^{0} \rightarrow \mu^{+} \mu^{-}\right)}$is the ratio of overall geometric and kinematic acceptances (including trigger and offline reconstruction efficiencies) between the signal and normalization modes. $B R\left(D^{0} \rightarrow \pi^{+} \pi^{-}\right)$is the PDG branching ratio of the normalization mode. We perform a blind analysis, where the selection cuts were optimized on a kinematically similar but independent set of events. We select events where the reconstructed $D^{0}$ candidates are matched with a soft pion track to reconstruct a $D^{*}$ candidate, and the mass difference between $D^{*}$ and $D^{0}$ is between 0.144 and 0.147 $\mathrm{GeV} / \mathrm{c}^{2}$.

To estimate the background we consider two sources: $D^{0} \rightarrow \pi^{+} \pi^{-}$and $D^{0} \rightarrow K^{ \pm} \pi^{\mp}$ decays where the pions(kaon) are misidentified as muons, and combinatorics. The overall pion misidentification probability is $1.3 \%$, which yields $0.22 \pm 0.02$ expected events from misidentified background. We estimate the combinatoric background from the high mass sideband, where both tracks are muons. This yields $1.5 \pm 0.7$ expected combinatoric background events. We find zero events in the search window of 1.84 to 1.884 $\mathrm{GeV} / \mathrm{c}^{2}$ (see Fig.2). The resulting limit on the branching ratio is

$$
\begin{aligned}
& B R\left(D^{0} \rightarrow \mu^{+} \mu^{-}\right)<2.4 \times 10^{-6} \text { at90\%C.L. } \\
& B R\left(D^{0} \rightarrow \mu^{+} \mu^{-}\right)<3.1 \times 10^{-6} \text { at95\%C.L. }
\end{aligned}
$$

This result improves the existing best limits from BEATRICE and E771 (see [5]), by almost a factor of 2 . 
4 Search for the FCNC Decay $B_{s}^{0} \rightarrow \mu^{+} \mu^{-}$

In the Standard Model, the branching ratio of $B_{s}^{0} \rightarrow \mu^{+} \mu^{-}$ is estimated to be $B R\left(B_{s}^{0} \rightarrow \mu^{+} \mu^{-}\right)=3.8 \times 10^{-9}[6]$. The best upper limit on $B R\left(B_{s}^{0} \rightarrow \mu^{+} \mu^{-}\right)$comes from CDF Run I, $B R\left(B_{s}^{0} \rightarrow \mu^{+} \mu^{-}\right)<2.6 \times 10^{-6}$ at the $95 \%$ C.L.[7] In many SUSY models this can be enhanced by one to three orders of magnitude (see for example[8]), which would make it observable in Run II.

The absolute number of expected $B_{s}^{0} \rightarrow \mu^{+} \mu^{-}$events is given by the expression

$$
N\left(B_{s}^{0} \rightarrow \mu^{+} \mu^{-}\right)=2 \sigma_{B_{s}^{0}} B R\left(B_{s}^{0} \rightarrow \mu^{+} \mu^{-}\right) \mathcal{L}_{\text {total }} \epsilon_{\text {total }} \alpha
$$

where $\sigma_{B_{s}^{0}}$ is the total $B_{s}^{0}$ production cross section in $p \bar{p}$ collisions, $\mathcal{L}_{\text {total }}$ is the total integrated luminosity, $\epsilon_{\text {total }}$ is the total efficiency (including trigger, reconstruction and analysis cut efficiencies), and $\alpha$ is the geometric and kinematic acceptance of the detector and trigger. For this analysis we use oppositely charged muons from a dimuon trigger which requires $2.7<M_{\mu \mu}<6.0 \mathrm{GeV} / \mathrm{c}^{2}$. The acceptance for this signature is estimated from the simulation, where we use events with transverse momentum of the b-quark $>6 \mathrm{GeV} / \mathrm{c}$ and rapidity between -1 and 1 . Trigger and reconstruction efficiencies are measured from the data. The analysis cut efficiencies are estimated from the simulation, which was validated by comparing the cut efficiencies of $B^{+} \rightarrow J / \Psi K^{+}$in data and simulation. For the total $B_{s}^{0}$ production cross section we assume $\sigma_{B_{s}^{0}}=\frac{1}{3} \sigma_{B_{d}^{0}}$, where $\sigma_{B_{d}^{0}}=3.6 \pm 0.72 \mu b$, as measured in Run I [9]. The error associated with the total $B_{s}^{0}$ production cross section is dominating the analysis. We select pairs of oppositely charged muons whose invariant mass is between 4.669 and $5.969 \mathrm{GeV} / \mathrm{c}^{2}$. The discriminating variables used to identify the signal are the invariant mass of the muon pair $M_{\mu \mu}$, the proper lifetime of the $B_{s}^{0}$ decay vertex, $c \tau$, the angular difference between the direction of the decay vertex and the $B_{s}^{0}$ flight direction, $\Delta \Phi$, and the isolation of the candidate $B_{s}^{0}$, ("Iso"). The background is estimated using the following expression,

$$
B g d=N_{S B}(c \tau, \Delta \Phi) R_{I s o} R_{m a s s}
$$

where $N_{S B}(c \tau, \Delta \Phi)$ is the number of events in the mass sidebands passing a particular set of $c \tau$ and $\Delta \Phi$ cuts, $R_{I s o}$ is the expected background rejection for a given Iso cut and $R_{\text {mass }}$ is the expected number of events in the signal mass-window given a number of surviving background events in the sideband regions. This method is valid since the only correlated variables are $\Delta \Phi$ and $c \tau$. Since mass and isolation are uncorrelated we can evaluate their rejection factors on samples with very loose $c \tau$ and $\Delta \Phi$ cuts, thus reducing their associated uncertainty. The resulting background is estimated to be $0.57 \pm 0.22$ events. We estimate residual background contributions from $B \rightarrow h^{+} h^{-}$ decays to be smaller than $10^{-9}$.

The resulting estimated sensitivity to $B_{s}^{0} \rightarrow \mu^{+} \mu^{-}$decays is

$$
B R\left(B_{s}^{0} \rightarrow \mu^{+} \mu^{-}\right)<2.5 \times 10^{-6}
$$

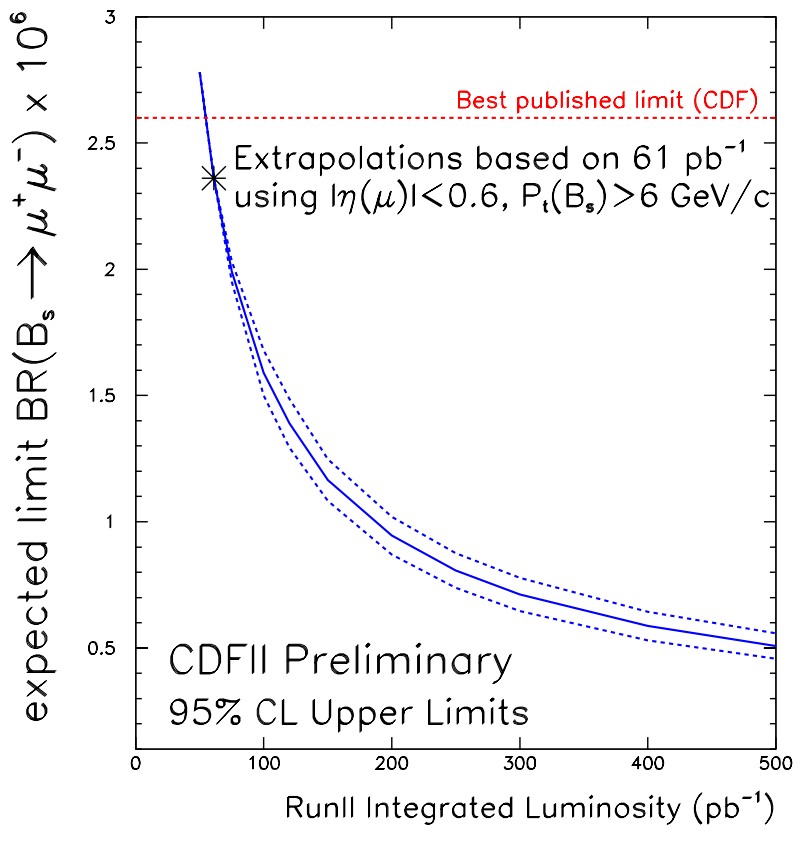

Fig. 3. The expected limit of $B R\left(B_{s}^{0} \rightarrow \mu^{+} \mu^{-}\right)$as a function of the integrated luminosity.

The analysis can also be performed for $B_{d}^{0} \rightarrow \mu^{+} \mu^{-}$decays and yields an estimated sensitivity of

$$
B R\left(B_{d}^{0} \rightarrow \mu^{+} \mu^{-}\right)<8.0 \times 10^{-7}
$$

Fig.3 shows the expected limit of $B R\left(B_{s}^{0} \rightarrow \mu^{+} \mu^{-}\right)$as a function of the integrated luminosity. The existing limit can be improved by a factor of 2 with the data currently on tape, and by a factor of $\sim 3$ within a year.

\section{References}

1. A.A.Petrov, hep-ph/0009160

2. FOCUS Collaboration, Phys.Lett.B 555, (2003) 167

3. CLEO Collaboration, hep-ex/0111024

4. G.Burdmann, E.Golowich, J.Hewett, S.Pakvasa, hep$\mathrm{ph} / 0112235 \mathrm{v} 2(2002)$

5. BEATRICE Collaboration, Phys.Lett.B 408, (1997) 469.

6. G.Buchalla and A.J.Buras, Nucl.Phys.B 412, (1994) 106.

7. CDF Collaboration, Phys.Rev. D 57, (1998) R3811.

8. A.Dedes, H.K.Dreiner, U.Nierste, P.Richardson, hep$\mathrm{ph} / 0207026$ (2002).

9. CDF Collaboration, Phys.Rev. D 65, (2002) 052005. 